\title{
FISIOTERAPIA NO FIBROEDEMA GELOIDE: ANÁLISE DE PERIÓDICOS NACIONAIS
}

\author{
PHYSIOTHERAPY IN FIBER EDEMA GELOIDE: ANALYSIS OF NATIONAL PERIODICALS
}

\author{
Lucas Lima Ferreira $^{\mathrm{a}^{*}}$, Camila Fernandes ${ }^{\mathrm{b}^{* *}}$, Simone Cavenaghi $^{* * *}$ \\ alucas_lim21@hotmail.com, bcamilaopg@hotmail.com, csicavenaghi@ig.com.br \\ "Universidade Estadual Paulista - Presidente Prudente (SP), Brasil \\ *Universidade Gama Filho - Rio de Janeiro (RJ), Brasil \\ ${ }^{* * *}$ Faculdade de Medicina de São José do Rio Preto - São José do Rio Preto (SP), Brasil
}

Data de entrega do artigo: 26/09/2013

Data de aceite do artigo: 28/04/2014

\section{RESUMO}

Introduçáo: $\mathrm{O}$ fibroedema geloide (FEG), popularmente conhecido como celulite, é uma alteração comum da topografia da pele, que acomete milhóes de mulheres no mundo. Por ser uma desordem multifatorial, o tratamento do FEG deve contemplar diferentes aspectos. Nesse contexto, a fisioterapia dermato-funcional (FDF) tem despontado como alternativa para o tratamento desta afecção, por meio de diversos recursos e técnicas específicas. Contudo, só um limitado número de estudos referentes ao tema tem sido publicado na literatura científica, com conclusôes contraditórias. Objetivo: Reunir estudos publicados em periódicos nacionais sobre a intervenção da FDF no FEG, a fim de propiciar uma atualização dos achados para a área. Métodos: Foi realizada uma revisão da literatura nacional, no período de 2002 a 2012, por meio das bases de dados LILACS e SciELO, utilizando os descritores fisioterapia "physiotherapy", estética "aesthetics" e celulite "cellulitis". Resultados: Foram encontrados 25 artigos dos quais apenas sete contemplaram os critérios de seleção e abordaram recursos da FDF no tratamento do FEG. Conclusão: Por meio desta revisão, verificou-se que as técnicas descritas na literatura nacional para o tratamento do FEG, utilizadas pela FDF, são o ultrassom terapêutico, associado ou não a agentes farmacológicos, a drenagem linfática manual, a endermologia e a vacuoterapia.

Palavras-chave: Celulite; modalidades de fisioterapia; estética.

\section{ABSTRACT}

Introduction: The fibroedema geloid (FEG), popularly known as cellulitis, is a common alteration of the topography of the skin that affects millions of women worldwide. Because it is a multifactorial disorder, the treatment of the FEG should contemplate different aspects. In this context, functional physiotherapy dermatology (FPD) has emerged as an alternative for the treatment of this disease through various resources and techniques. However, only a limited number of studies on the subject have been published in the scientific literature with contradictory conclusions. Objective: To gather studies published in national journals on the intervention of the FPD and FEG in order to provide an update of the findings for the area. Methods: We conducted a review of national literature in the period of 2002 to 2012, through the databases LILACS and SciELO using the keywords physiotherapy, aesthetic and cellulitis. Results: We found 25 articles of which only seven beheld the selection criteria and addressed the FPD resources in the treatment of FEG. Conclusion: Through this study it was found that the techniques described in the national literature for the treatment of FEG, used by the FPD, are therapeutic ultrasound, alone or combined with pharmacological agents, the manual lymphatic drainage, endermologie and the vacuum therapy.

Keywords: Cellulitis; physical therapy modalities; esthetics. 


\section{Introdução}

O fibroedema geloide (FEG), popularmente conhecido como "celulite", é uma alteração comum da topografia da pele, indesejável esteticamente, que acomete milhóes de mulheres no mundo ${ }^{1-3}$. Este se manifesta por contornos irregulares na pele a partir da puberdade ${ }^{1,2,4-6}$. É definido como uma disfunção metabólica localizada, do tecido subcutâneo e da derme, a qual provoca alteração na forma corporal, causada pelo excesso de tecido adiposo retido no septo fibroso ${ }^{1,5}$ e por projeçóes deste na derme $e^{2}$.

O termo celulite, que significa condição de inflamação do tecido celular, foi descrito pela primeira vez na França, por volta de $1920^{1,4,7}$, e desde entâo vem sendo utilizado para descrever a aparência ondulada e irregular da pele, com aspecto de casca de laranja ou queijo tipo cottage ${ }^{8}$.

Embora não exista morbidade ou mortalidade associada à celulite, ou seja, não se trata de doença, ela permanece como preocupação estética frequentemente importante ${ }^{9,10}$. O FEG é muito mais prevalente nas mulheres e tende a ocorrer nas áreas em que a gordura está sob a influência do estrógeno, como quadris, coxas e nádegas, mas também pode ser encontrado em mamas, parte inferior do abdômen, braços e nuca, áreas em que o padrão feminino de deposiçáo do tecido adiposo é observado ${ }^{8,10}$.

A etiologia do FEG é desconhecida, mas uma variedade de causas parece contribuir para o seu desenvolvimento, incluindo fatores estruturais, circulatórios, hormonais e inflamatórios ${ }^{8}$. Alguns autores ${ }^{8}$ descrevem três hipóteses etiológicas que se baseiam em alteraçōes anatômicas e hormonais, microcirculação e processo inflamatório crônico.

Em termos metodológicos, Hexsel et al. ${ }^{11}$ desenvolveram uma classificação da celulite de forma objetiva por meio de escalas fotonuméricas, composta de cinco variáveis: número de depressóes evidentes; profundidade das depressóes visíveis; aparência morfológica das alteraçóes de superfície da pele; grau de flacidez ou frouxidão cutânea; classificaçáo da escala de Nürenberger e Müller ${ }^{12}$; em que a soma final da pontuação classifica o indivíduo em uma de três categorias de gravidade: leve (1-5 pontos), moderada (6-10 pontos) e grave (11-15 pontos).

Por ser uma desordem multifatorial, o tratamento do FEG deve contemplar diferentes aspectos e se direcionar a tratar a fibrose, a flacidez muscular, o acúmulo de gordura, a tonificação cutânea e o edema tecidual ${ }^{13}$. Uma variedade de terapias tem sido proposta para o tratamento do FEG, objetivando a perda de peso graças à diminuição da gordura subcutânea, reduzindo a aparência da casca de laranja, o que leva à procura de métodos terapêuticos para reduçáo de medidas de maneira mais eficaz $^{14}$.

Nesse contexto, a fisioterapia dermato-funcional (FDF) tem despontado como alternativa para o tratamento do FEG, por meio de diversos recursos e técnicas específicas ${ }^{15}$. Porém, só um limitado número de estudos referentes a esse assunto tem sido publicado na literatura científica, tendo muitos deles chegado a conclusôes contraditórias ${ }^{16}$. Além disso, não foram encontrados, até o momento, trabalhos na literatura nacional que sistematizassem a aplicação de FDF no FEG, dessa forma, o presente estudo tem o objetivo de reunir estudos publicados em periódicos nacionais sobre a intervenção da FDF na celulite, a fim de propiciar uma atualização dos achados para a área.

\section{Métodos}

\section{Estratégia de busca}

O delineamento metodológico deste estudo caracterizou-se por uma revisão de literatura por meio de artigos nacionais selecionados a partir de consultas às bases de dados SciELO e LILACS, no período de 2002 a 2012. Para a busca, foram utilizados os cruzamentos das palavras-chave: fisioterapia "physiotherapy" e estética "aesthetics" por meio do operador booleano "and", com o descritor celulite "cellulitis"; as quais foram definidas com base nos Descritores em Ciências da Saúde (DeCS) e seus correspondentes na língua inglesa $(\mathrm{MeSH})$.

Outra estratégia utilizada foi a busca manual nas listas de referências dos estudos selecionados para complementação da pesquisa. Todas as etapas da busca foram realizadas por somente um avaliador.

\section{Critérios de inclusão e exclusão}

Foram incluídos estudos publicados nos últimos dez anos, nas línguas inglesa e portuguesa, com seres humanos portadores de FEG submetidos a algum tipo de intervenção fisioterapêutica. Foram incluídos estudos longitudinais, randomizados e não randomizados.

Resumos de dissertaçóes ou teses acadêmicas, trabalhos de conclusão de curso de graduação e artigos de periódicos internacionais foram excluídos.

\section{Estratégia de seleção}

Para a seleção dos artigos, inicialmente foi realizada a triagem dos títulos relacionados ao tema em questão. 
Esta seleção foi baseada nos títulos que abordassem como ideia principal: o papel da FDF no tratamento da celulite (FEG). Ao final da busca, foram excluídos os títulos repetidos, já que esta foi realizada em diversas bases de dados. Em seguida, foi feita a leitura detalhada dos resumos dos artigos a fim de selecionar aqueles que abordassem exclusivamente técnicas de fisioterapia sobre o FEG. Excluídos os resumos que não versavam sobre o tema, os textos completos foram avaliados e os que não se enquadravam nos critérios de exclusão foram inclusos como resultado final da busca.

\section{Análise dos dados}

Os dados foram analisados de forma qualitativa e apresentados na forma de tabela com a descrição das seguintes características: autor, tipo de estudo, características, objetivos do estudo, protocolo fisioterapêutico e resultados encontrados.

\section{Resultados}

A busca nas bases de dados resultou em 25 artigos, cinco publicados na base SciELO e 20 na base Lilacs, que foram submetidos às estratégias de seleção para elegibilidade no presente estudo (Figura 1).

Figura 1: Fluxograma da estratégia de seleção dos artigos

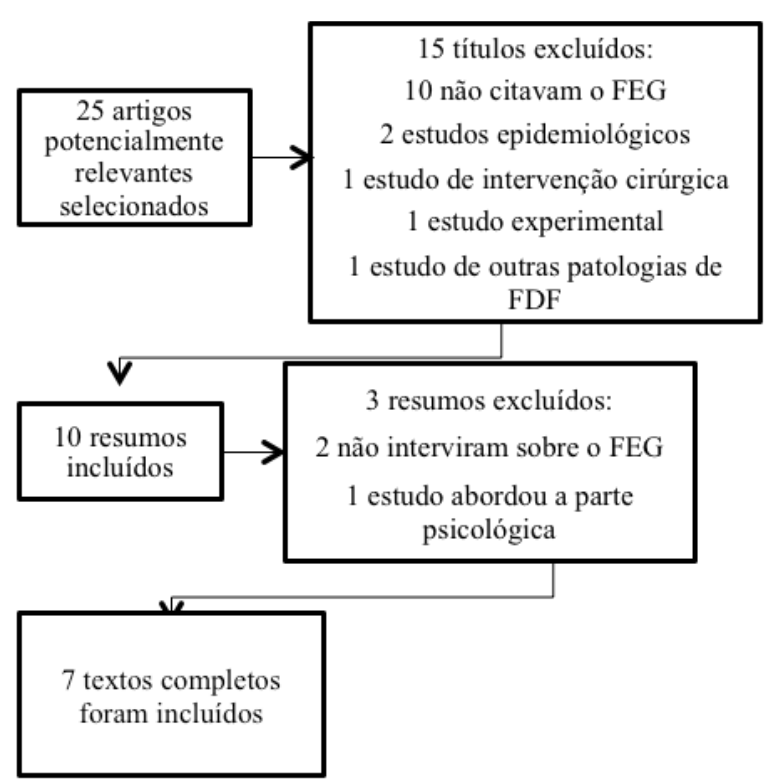

Abreviaturas: $\mathrm{FEG}=$ fibroedema geloide; $\mathrm{FDF}=$ fisioterapia dermato-funcional.
Como resultado final, foram identificados sete artigos, um ensaio clínico randomizado ${ }^{17}$, três revisóes de literatura ${ }^{18,19,21}$, um estudo piloto ${ }^{20}$, um estudo descritivo $^{22}$, e um estudo clínico ${ }^{23}$, que aplicaram diferentes técnicas fisioterapêuticas para o tratamento do FEG (tabela 1). Entre os artigos de revisão: um discorreu sobre o ultrassom terapêutico (UST) ${ }^{18}$, outro sobre endermologia $^{19}$ e um sobre vacuoterapia ${ }^{21}$ no FEG. Um estudo desenvolveu um protocolo de avaliação fisioterapêutico específico para pacientes com $\mathrm{FEG}^{22}$; e três estudos analisaram os efeitos do UST sobre a celulite: um o comparou com a eletrolipoforese ${ }^{17}$, outro aplicou o UST por meio da fonoforese ${ }^{20}$ e um terceiro artigo associou o UST às manobras de drenagem linfática manual (DLM).

Quatro estudos foram de intervençáo ${ }^{17,20,22,23}$, nos quais, o tamanho amostral variou de cinco a 30 sujeitos, e, no total, esses estudos envolveram 67 indivíduos, todos do gênero feminino, com idades que variaram entre 17 a 69 anos. Nenhum destes estudos utilizou grupo controle.

\section{Discussão}

A presente revisão verificou a escassez de estudos na literatura nacional sobre a FDF no FEG, fato evidenciado pela inclusão de apenas sete artigos científicos como resultado final das buscas. Com base nas publicações encontradas, pode-se constatar que a técnica mais utilizada por fisioterapeutas brasileiros no tratamento da celulite tem sido o UST, associado ou não a agentes farmacológicos, porém outras técnicas, como a DLM, a endermologia e a vacuoterapia, também foram investigadas no tratamento do FEG.

Machado et $a .^{17}$ avaliaram os efeitos da aplicação do UST e da eletrolipoforese (ELF) em 22 voluntárias sedentárias, que faziam uso de anticoncepcional, com FEG graus I e/ou II na região glútea. As voluntárias foram divididas em dois grupos de 11 pacientes, nas quais um recebeu tratamento com UST e o outro com ELF durante dez sessóes. Os autores observaram melhora no aspecto visual do FEG, por meio da avaliação fotográfica e na satisfação pessoal em ambos os grupos, sem diferenças estatisticamente significativas para a redução de medidas antropométricas e de bioimpedância. Tal fato pode ser explicado pelos efeitos terapêuticos localizados e discretos dos recursos utilizados.

Um estudo sobre a aplicaçáo do UST no tratamento das alteraçóes decorrentes do FEG realizou uma revisão de literatura e encontrou dez artigos, publicados na década de 1990, que indicaram que o UST foi eficaz na amenização e diminuição do quadro clínico da celulite nas principais regiôes corporais que acometem 
Tabela 1: Características dos estudos que aplicaram modalidades de fisioterapia no FEG

\begin{tabular}{|c|c|c|c|c|c|}
\hline Autor & $\begin{array}{l}\text { Tipo de } \\
\text { estudo }\end{array}$ & Características & Objetivos & Protocolo fisioterapêutico & Resultados \\
\hline $\begin{array}{l}\text { Machado } \\
\text { et al. }{ }^{17}\end{array}$ & $\begin{array}{c}\text { Ensaio } \\
\text { clínico } \\
\text { prospectivo } \\
\text { randomizado }\end{array}$ & $\begin{array}{l}22 \text { voluntárias, } \\
\text { idade entre } 18 \text { e } 35 \\
\text { anos, sedentárias, } \\
\text { FEG grau I e II em } \\
\text { glúteos, usuárias } \\
\text { de contraceptivos }\end{array}$ & $\begin{array}{l}\text { Avaliar os efeitos } \\
\text { do UST e da ELF } \\
\text { no tratamento } \\
\text { das alteraçóes } \\
\text { decorrentes do } \\
\text { FEG. }\end{array}$ & $\begin{array}{c}\text { Dez sessões, três vezes por } \\
\text { semana, com UST em } 11 \\
\text { voluntárias e com ELF em } 11 \\
\text { voluntárias; } \\
\text { UST - modo contínuo, } \\
\text { frequência de } 3 \mathrm{MHz} \text {, intensi- } \\
\text { dade } 1,0 \mathrm{Wcm}^{2} \text {, tempo de } 2 \\
\text { min. para cada área de } 10 \mathrm{~cm} \\
\text { (glúteos divididos em quatro } \\
\text { quadrantes, bilateralmente); } \\
\text { ELF - quatro canais de saída de } \\
\text { corrente, eletrodos de placa de } \\
\text { silicone, } 40 \text { min. de aplicação } \\
\text { epicutânea. }\end{array}$ & $\begin{array}{c}\text { Não houve diferença } \\
\text { significante na perimetria, } \\
\text { adipometria e bioimpedância } \\
\text { após os tratamentos instituídos. } \\
\text { Na avaliaçáo fotográfica, } \\
\text { houve melhora no aspecto } \\
\text { visual do FEG em } 68,18 \% \\
\text { das participantes. A satisfaçáo } \\
\text { pessoal aumentou em ambos os } \\
\text { grupos. }\end{array}$ \\
\hline $\begin{array}{l}\text { Menezes } \\
\text { et al. }{ }^{18}\end{array}$ & $\begin{array}{l}\text { Revisão de } \\
\text { literatura }\end{array}$ & $\begin{array}{l}\text { Dez artigos sobre } \\
\text { FEG associado ao } \\
\text { UST, publicados } \\
\text { entre } 1992 \text { a } 2004\end{array}$ & $\begin{array}{l}\text { Avaliar o benefício } \\
\text { e a eficácia do UST } \\
\text { para o tratamento } \\
\text { do FEG. }\end{array}$ & & $\begin{array}{c}\text { Os estudos selecionados } \\
\text { indicaram que o tratamento } \\
\text { do FEG com UST foi eficaz na } \\
\text { amenizaçáo e diminuição do } \\
\text { quadro nas regiōes de glúteos, } \\
\text { coxas e abdômen. }\end{array}$ \\
\hline $\begin{array}{l}\text { Sant'Ana } \\
\text { et al. }{ }^{19}\end{array}$ & $\begin{array}{l}\text { Revisão de } \\
\text { literatura }\end{array}$ & $\begin{array}{l}\text { Oito artigos sobre } \\
\text { endermologia no } \\
\text { tratamento do } \\
\text { FEG, publicados } \\
\text { entre } 1998 \text { a } 2006\end{array}$ & $\begin{array}{l}\text { Revisar aspectos } \\
\text { fisiopatológicos } \\
\text { do FEG e seu } \\
\text { tratamento com } \\
\text { endermologia. }\end{array}$ & & $\begin{array}{l}\text { Os estudos têm demonstrado } \\
\text { que a endermologia é eficaz no } \\
\text { tratamento do FEG, diminuição } \\
\text { e redistribuição da gordura } \\
\text { localizada, sem relatos de efeitos } \\
\text { adversos. }\end{array}$ \\
\hline $\begin{array}{l}\text { Federico } \\
\text { et al. }{ }^{20}\end{array}$ & $\begin{array}{l}\text { Estudo } \\
\text { piloto }\end{array}$ & $\begin{array}{l}\text { Cinco voluntárias, } \\
\text { idade entre } 20 \text { e } \\
30 \text { anos, FEG grau } \\
\text { II em região glútea }\end{array}$ & $\begin{array}{c}\text { Levantar resultados } \\
\text { preliminares acerca } \\
\text { da eficácia do uso } \\
\text { do UST, associado } \\
\text { à fonoforese, na } \\
\text { redução do FEG } \\
\text { localizado. }\end{array}$ & $\begin{array}{c}16 \text { sessóes, quatro vezes por } \\
\text { semana, com UST modulado } \\
\text { da seguinte forma: } \\
\text { UST - modo contínuo, } \\
\text { frequência de } 3 \mathrm{MHz} \text {, inten- } \\
\text { sidade } 1,5 \mathrm{Wcm}^{2} \text { (transdutor } \\
\left.\text { com ERA de } 4 \mathrm{~cm}^{2}\right), 1,1 \mathrm{Wcm}^{2} \\
(\text { transdutor com ERA de } \\
\left.8,5 \mathrm{~cm}^{2}\right) \text { tempo de } 1 \text { min. para } \\
\text { cada área de acordo com a ERA } \\
\text { do transdutor utilizado (glúteos } \\
\text { divididos em dois quadrantes, } \\
\text { unilateralmente); } \\
\text { Fonoforese - acoplamento à } \\
\text { base de hera, centella asiática e } \\
\text { castanha da índia. }\end{array}$ & $\begin{array}{l}\text { A fonoforese mostrou-se eficaz } \\
\text { no tratamento da celulite } \\
\text { somente em uma das cinco } \\
\text { voluntárias envolvidas na } \\
\text { pesquisa. }\end{array}$ \\
\hline $\begin{array}{l}\text { Bacelar e } \\
\text { Vieira }^{21}\end{array}$ & $\begin{array}{l}\text { Revisão de } \\
\text { literatura }\end{array}$ & $\begin{array}{l}\text { Nove artigos sobre } \\
\text { tratamento do } \\
\text { FEG, publicados } \\
\text { entre } 1998 \text { e } 2005\end{array}$ & $\begin{array}{l}\text { Verificar a eficácia } \\
\text { da vacuoterapia } \\
\text { no tratamento do } \\
\text { FEG. }\end{array}$ & & $\begin{array}{l}\text { A vacuoterapia é de grande im- } \\
\text { portância na reversão do quadro } \\
\text { de FEG, por incrementar a } \\
\text { circulação sanguínea e linfática, } \\
\text { melhorar a maleabilidade do } \\
\text { tecido conjuntivo, diminuir as } \\
\text { aderências e a fibrose. }\end{array}$ \\
\hline
\end{tabular}

continua... 
Tabela 1: Continuação

\begin{tabular}{|c|c|c|c|c|c|}
\hline Autor & $\begin{array}{l}\text { Tipo de } \\
\text { estudo }\end{array}$ & Características & Objetivos & Protocolo fisioterapêutico & Resultados \\
\hline $\begin{array}{c}\text { Meyer } e t \\
a l^{22}\end{array}$ & $\begin{array}{c}\text { Estudo } \\
\text { descritivo }\end{array}$ & $\begin{array}{c}30 \text { voluntárias, } \\
\text { idade entre } 20 \text { e } \\
69 \text { anos, FEG grau } \\
\text { I e II }\end{array}$ & $\begin{array}{c}\text { Desenvolver } \\
\text { e aplicar um } \\
\text { instrumento de } \\
\text { coleta de dados, que } \\
\text { permita avaliar o } \\
\text { grau do FEG e os } \\
\text { níveis de alteraçōes } \\
\text { sensitivas. }\end{array}$ & $\begin{array}{c}\text { Desenvolvimento do PAFEG: } \\
\text { Validade de face - consultados } \\
\text { cinco fisioterapeutas com } \\
\text { questionário sobre o conteúdo } \\
\text { do PAFEG; } \\
\text { Validade de conteúdo - } \\
\text { consultados três profissionais } \\
\text { que atuam em FDF com } \\
\text { questionário sobre o conteúdo } \\
\text { do PAFEG; } \\
\text { Avaliação de sensibilidade - } \\
\text { teste de preensão e teste dos } \\
\text { monofilamentos nas pacientes } \\
\text { com FEG; } \\
\text { Aplicação do PAFEG nas } \\
\text { pacientes com FEG. }\end{array}$ & $\begin{array}{l}\text { Observou-se que o PAFEG é } \\
\text { de fácil aplicação e abrange } \\
\text { todos os aspectos do FEG, } \\
\text { estando apto a ser utilizado } \\
\text { em avaliaçóes de pacientes } \\
\text { portadoras desta afecção. }\end{array}$ \\
\hline $\begin{array}{c}\text { Almeida et } \\
\text { al. }{ }^{23}\end{array}$ & $\begin{array}{l}\text { Estudo } \\
\text { clínico }\end{array}$ & $\begin{array}{l}\text { Dez voluntárias } \\
\text { do sexo feminino, } \\
\text { idade média de } 24 \\
\pm 5,33 \text { anos, com } \\
\text { FEG graus I e III } \\
\text { em região glútea e } \\
\text { posterior de coxa }\end{array}$ & $\begin{array}{c}\text { Analisar o efeito da } \\
\text { DLM associada ao } \\
\text { US de } 3 \mathrm{MHz} \text { como } \\
\text { medidas terapêuti- } \\
\text { cas no tratamento } \\
\text { de mulheres com } \\
\text { FEG. }\end{array}$ & $\begin{array}{l}\text { Técnicas de DLM de Leduc por } \\
\qquad 60 \text { min.; } \\
\text { UST com dose de } 0,6 \mathrm{~W} / \mathrm{cm}^{2} \text {, } \\
\text { frequência de } 3 \mathrm{MHz}, \text { modo } \\
\text { contínuo, duraçáo de } 24 \text { min.; } \\
\text { dez sessōes, duas vezes por } \\
\text { semana. }\end{array}$ & $\begin{array}{l}\text { Verificou-se diferença estatística } \\
\text { significante no grau do FEG na } \\
\text { região glútea e na satisfaçáo das } \\
\text { pacientes quando comparados } \\
\text { os locais antes e após } \\
\text { tratamento. }\end{array}$ \\
\hline
\end{tabular}

Abreviaturas: FEG = fibroedema geloide; UST = ultrassom terapêutico; ELF = eletrolipoforese; $\mathrm{MHz}=$ mega-hertz; $\mathrm{W}=$ watts; $\mathrm{cm}^{2}$ = centímetro quadrado; min. = minutos; PAFEG = protocolo de avaliação do fibroedema geloide; FDF = fisioterapia dermato-funcional; DLM = drenagem linfática manual.

as mulheres ${ }^{18}$. Os autores atribuem tais resultados aos efeitos fisiológicos do UST, caracterizados pela ação tixotrópica sobre géis, despolimerização da substância fundamental, deslocamento de íons, aumento da permeabilidade das membranas, melhor reabsorção de líquidos, aperfeiçoamento da irrigação sanguínea e linfática, aumento da produção e melhora de orientação das fibras colágenas do tecido conjuntivo.

Outro estudo de revisão ${ }^{19}$ analisou os efeitos da endermologia no tratamento do FEG, por meio de estudos publicados no final da década de 1990. A endermologia é uma técnica de massagem que consiste de roletes com pressão positiva em conjunto com pressão negativa aplicados na pele e tecido subcutâneo e, segundo as autoras, se desponta como uma proposta de tratamento não invasivo para FEG, por causar diminuição e redistribuição da gordura localizada.

Federico et al. ${ }^{20}$ realizaram um estudo piloto com cinco voluntárias adultas jovens, com FEG grau II em região glútea, aplicando UST associado à fonoforese em 16 sessões. A fonoforese consiste na habilidade do UST em incrementar a penetração de agentes farmacológicos ativos através da pele. Esta foi realizada empregando substância de acoplamento em forma de gel, composta de extratos de hera, centella asiática e castanha da índia. As autoras verificaram que a técnica foi eficaz somente em uma voluntária, e atribuem tal fato ao tempo destinado ao tratamento (4 semanas), que foi insuficiente para que houvesse uma ação do princípio ativo, assim como dos efeitos próprios do UST, além disso, também salientam que o tamanho reduzido da amostra pode ter comprometido os resultados.

A vacuoterapia é uma técnica rítmica de dobramento e desdobramento com sucção do panículo adiposo, que utiliza ventosa com roletes em conjunto com a pressão negativa da sucção ${ }^{21}$. A eficácia dessa técnica no tratamento do FEG foi alvo de um estudo de revisão, que analisou nove publicaçóes sobre o tema e verificou que a vacuoterapia é de grande importância na reversão do quadro de FEG, por incrementar a circulação sanguínea e linfática, melhorar a maleabilidade do tecido conjuntivo, diminuir as aderências e a fibrose ${ }^{21}$.

Já Meyer et al..$^{22}$ desenvolveram e aplicaram um protocolo de avaliaçáo do FEG, denominado PAFEG, em 
30 voluntárias com celulite grau I e oito fisioterapeutas. Os autores observaram que o PAFEG é de fácil aplicação e, com base nos testes feitos nos fisioterapeutas, permite classificar, de forma adequada e objetiva, o grau do FEG, assim como os níveis das alteraçóes sensitivas quando estas estiverem presentes. Abrange todos os aspectos do FEG, estando apto a ser utilizado em avaliaçóes fisioterapêuticas de pacientes portadoras desta afecção.

Almeida et al. ${ }^{23}$ analisaram os efeitos da DLM associada ao UST no tratamento do FEG graus I e III em dez mulheres jovens. Os autores observaram diferença significativa no grau do FEG e na satisfação das pacientes na comparação entre antes e após o tratamento, e atribuem tais resultados aos efeitos fisiológicos da aplicação do UST, que são o aquecimento dos tecidos biológicos levando à intensificação do fluxo sanguíneo, da atividade metabólica e ao aumento da extensibilidade do colágeno; e à DLM, que é realizada por meio de movimentos de bombeamento com pressôes suaves e rítmicas, estimulando o fluxo linfático e reduzindo o edema.

Quatro dos estudos analisados nesta revisão analisaram os efeitos da aplicação do UST sobre as alteraçôes decorrentes do FEG, em indivíduos do gênero feminino. A principal aplicaçáo do UST nos tecidos biológicos envolve a produção de hiperemia, o aumento de leucócitos e anticorpos, a açáo espasmolítica, a ação trófica, a analgesia, a antiflogística, o aumento da extensibilidade dos tendóes, a destruiçáo de macromoléculas, a reabsorção de edemas, a eliminação de macronódulos e do aspecto de casca de laranja no FEG, a correção da isquemia em áreas lipodistróficas, o aumento do intercâmbio iônico intercelular e a melhora do metabolismo lipídico com o aumento da lipólise ${ }^{17,24}$.

Em suma, os estudos analisados nesta revisão demonstraram diferentes técnicas utilizadas pela fisioterapia para o tratamento do FEG, demonstrando o arsenal terapêutico de que dispóe essa especialidade para tratar tal disfunçáo, caracterizando o fisioterapeuta como o profissional mais adequado para atuar junto às portadoras desta afecção.

\section{Conclusão}

A presente investigaçáo verificou que as técnicas descritas na literatura nacional para o tratamento do FEG, utilizadas pela fisioterapia dermato-funcional, são o UST, associado ou não a agentes farmacológicos, a DLM, a endermologia e a vacuoterapia. Contudo, a análise metodológica das publicaçôes encontradas demonstrou a falta de padronizaçáo das técnicas e métodos de avaliaçáo dos resultados obtidos, dificultando inferências a cerca dos níveis de evidência de tais recursos.
Constatou-se, ainda, a escassez de estudos sobre o tema publicados até o momento nos periódicos de circulação brasileira, o que sucinta a necessidade de novas investigaçóes sobre o tema, com desenhos metodológicos adequados.

\section{Referências}

1. Rossi AB, Vergnanini AL. Cellulite: a review. J Eur Acad Dermatol Venereol. 2000; 14(4): 251-62.

2. Terranova F, Berardesca E, Maibach H. Cellulite: nature and aethiopathogenesis. Int J Cosmet Sci. 2006; 28(3): 157-67.

3. Sadick N, Magro C. A study evaluating the safety and efficacy of the Velasmooth ${ }^{\mathrm{TM}}$ system in the treatment of the cellulite. J Cosmet Laser Ther. 2007; 9: 15-20.

4. Avram MM. Cellulite: a review of its physiology and treatment. J Cosmet Laser Ther. 2004; 6(4): 181-5.

5. Alster TS, Tanzi EL. Cellulite treatment using a novel combination radiofrequency, infrared light and mechanical tissue manipulation device. J Cosmet Laser Ther. 2005; 7(2): 81-5.

6. Mirrashed F, Sharp JC, Krause V, Morgan J, Tomanek B. Pilot study of dermal and subcutaneous fat structures by MRI in individuals who differ in gener, BMI, and cellulite grading. Skin Res Technol. 2004; 10(3): 161-8.

7. Rona C, Carrera M, Berardesca E. Testing anticellulite products. Int J Cosmet Sci. 2006; 28: 169-73.

8. Afonso JPJM, Tucunduva TCM, Pinheiro MVB, Bagatin E. Celulite: artigo de revisão. Surg Cosmet Dermatol. 2010; 2(3): 214-19.

9. Hexsel DM, Mazzucco R. Subcision: a treatment for cellulite. Int J Dermatol. 2000; 39: 539-44.

10. Machado AFP, Tacani RE, Schwartz J, Liebano RE, Ramos JLA, Frare T. Incidência de fibroedema geloide em mulheres caucasianas jovens. Arq Bras Ciên Saúde. 2009; 34(2): 80-6.

11. Hexsel DM, Dal'Forno T, Hexsel CL. A validated photonumeric cellulite severity scale. J Eur Acad Dermatol Venereol. 2009; 23(5): 523-8.

12. Nurnberger F, Muller G. So-called cellulite: an invented disease. J Dermatol Surg Oncol.1978; 4(3): 221-9.

13. Angelino BS. Fibroedema geloide subcutâneo: qué conocemos de esta entidad clínica? Folia Dermatol Peru. 2003; 14(1): 38-42.

14. Draelos ZD, Marenus KD. Cellulite: etiology andpurported treatment. Dermatol Surg. 1997; 23(12): 1177-81.

15. Milani GB, Amado-João SM, Farah EA. Fundamentos da fisioterapia dermato-funcional: revisão de literatura. Fisioter Pesq. 2006; 13(1): 37-43.

16. Terranova F, Berardesca E, Maibach I. Cellulite: nature and etiopathogenesis. Int J Cosmet Sci. 2006; 28(3): 157-67.

17. Machado GC, Vieira RB, de Oliveira NML, Lopes CR. Análise dos efeitos do ultrassom terapêutico e da 
eletrolipoforese nas alteraçóes decorrentes do fibroedema geloide. Fisioter Mov. 2011; 24(3): 471-9.

18. Menezes RC, Silva SG, Ribeiro ER. Ultrassom no tratamento do fibroedema geloide. Rev Inspirar. 2009; 1(1): 10-4.

19. Sant'Ana EMC, Marqueti RC, Leite VL. Fibroedema geloide (celulite): fisiopatologia e tratamento com endermologia. Fisioter Espec. 2007; 1(1): 30-5.

20. Federico MR, Gomes SVC, Melo VC, Martins RB, Lauria MC, Moura RL, et al. Tratamento de celulite (paniculopatia edemato fibroesclerótica) utilizando fonoforese com substância acoplante à base de hera, centella asiática e castanha da índia. Fisioter Ser. 2006; 1(1): 6-10.

21. Bacelar VCF, Vieira MES. Importância da vacuoterapia no tratamento do fibroedema geloide. Fisioter Bras. 2006; $7(6): 440-3$.
22. Meyer PF, Lisboa FL, Alves MCR, Avelino MB. Desenvolvimento e aplicação de um protocolo de avaliação fisioterapêutica em pacientes com fibroedema geloide. Fisioter Mov. 2005; 18(1): 75-83.

23. Almeida AF, Brandão DSM, Silva JC, Oliveira RGCQ, Araújo RC, Pitangui ACR. Avaliaçáo do efeito da drenagem linfática manual e do ultrassom no fibroedema geloide. Rev Bras Ciên Saúde. 2011; 9(28): 31-7.

24. Durigan JLQ, Cancelliero KM, Reis MS, Dias CNK, Graciotto DR, da Silva CA, et al. Mecanismos de interação do ultra-som terapêutico com tecidos biológicos. Rev Fisioter Brasil. 2006; 7(2): 142-8. 